\title{
Trefoil factor 1 inhibits epithelial-mesenchymal transition of pancreatic intraepithelial neoplasm
}

\author{
Junpei Yamaguchi, ${ }^{1}$ Yukihiro Yokoyama, ${ }^{1}$ Toshio Kokuryo, ${ }^{1}$ Tomoki Ebata, ${ }^{1}$ Atsushi Enomoto, ${ }^{2}$ and Masato Nagino \\ 'Division of Surgical Oncology, Department of Surgery, and 2Department of Pathology, Nagoya University Graduate School of Medicine, Nagoya, Aichi, Japan.
}

\begin{abstract}
The tumor-suppressive role of trefoil factor family (TFF) members in gastric carcinogenesis has been suggested, but their significance and mechanisms in other digestive diseases remain elusive. To clarify the role of TFF1 in pancreatic carcinogenesis, we performed IHC on human samples, transfected siRNA against TFF1 into pancreatic cancer cell lines, and employed mouse models in which PanIN development and loss of TFF1 occur simultaneously. In human samples, the expression of TFF1 was specifically observed in pancreatic intraepithelial neoplasm (PanIN), but was frequently lost in the invasive component of pancreatic ductal adenocarcinoma (PDAC). When the expression of TFF1 was suppressed in vitro, pancreatic cancer cell lines showed enhanced invasive ability and features of epithelial-mesenchymal transition (EMT), including upregulated Snail expression. TFF1 expression was also observed in PanIN lesions of Pdx-1 Cre; LSL-KRAS ${ }^{C 12 D}(\mathrm{KC})$ mice, a model of pancreatic cancer, and loss of TFF1 in these mice resulted in the expansion of PanIN lesions, an EMT phenotype in PanIN cells, and an accumulation of cancer-associated fibroblasts (CAFs), eventually resulting in the development of invasive adenocarcinoma. This study indicates that the acquisition of TFF1 expression is an early event in pancreatic carcinogenesis and that TFF1 might act as a tumor suppressor to prevent EMT and the invasive transformation of PanIN.
\end{abstract}

\section{Introduction}

Pancreatic ductal adenocarcinoma (PDAC) is one of the most lethal malignancies worldwide (1), partly because it is usually diagnosed at an advanced stage, with metastasis and extensive tumor invasion into other organs. It is of great importance to detect PDAC in its early stage and to understand the mechanisms by which pancreatic tumor cells obtain the ability to invade and metastasize. Epithelial-mesenchymal transition (EMT) has been recognized as the process by which tumor cells transform from an epithelial phenotype to a mesenchymal phenotype, and it is supposed to be a prerequisite for the invasion and dissemination of tumor cells (2). Although metastasis is thought to be the latest event in malignant tumors, recent studies have suggested that pancreatic tumor cells undergo EMT in their early stage before tumor formation $(3,4)$. There are 2 types of premalignant lesions of the pancreas: pancreatic intraepithelial neoplasm (PanIN) (5, 6) and intraductal papillary mucinous neoplasm (IPMN) $(7,8)$. These premalignant lesions already have somatic mutations in the KRAS oncogene, even in their early stages (PanIN-1 or lowgrade IPMN) $(9,10)$, suggesting that KRAS activation is an early event of pancreatic carcinogenesis. In genetic mouse models, KRAS activation in the pancreas indeed results in the development of PanIN; however, this PanIN rarely develops into invasive carcinoma. Thus, PanIN seems to require additional genetic changes to undergo EMT, but the crucial genes for early EMT have not been identified.

Conflict of interest: The authors have declared that no conflict of interest exists Submitted: September 28, 2017; Accepted: May 24, 2018.

Reference information: J Clin Invest. 2018;128(8):3619-3629.

https://doi.org/10.1172/JCI97755.
The trefoil factor family (TFF) is a group of small peptides with a characteristic 3-leafed structure that are abundantly expressed and secreted in the gastrointestinal mucosa. Three TFF members have been identified in mammals and are coexpressed with gastrointestinal mucins. TFF1 and MUC5AC are colocalized in foveolar cells of the stomach, TFF2 and MUC6 are colocalized in the fundus and deep antral gland of the stomach, and TFF3 and MUC2 are colocalized throughout the large and small bowel mucosa $(11,12)$. Based on the observation that the expression of TFFs increases in damaged mucosa, studies of TFFs have primarily focused on the repair of damaged gastrointestinal mucosa (restitution) (13-15). In addition to this classic role of TFFs, recent reports indicate that TFFs can also act as tumor suppressors in gastric carcinogenesis (16-19). In pancreatic carcinogenesis, PanIN and IPMN share a distinguishing characteristic: abundant expression of gastrointestinal mucins, such as MUC2, MUC5AC, and MUC6, depending on the phenotypes of the lesions (20-22). This finding suggests not only that gastrointestinal metaplasia is the first step of pancreatic carcinogenesis, but also that pancreatic tumorigenesis might share genetic mechanisms with gastric tumorigenesis. Given that TFFs might function as tumor suppressors in the stomach, one can assume that they may also inhibit oncogenesis in the pancreas.

In this study, we show that the acquisition of TFF1 expression occurs early in PanIN development, but TFF1 expression is frequently lost in the invasive component of PDAC. Loss of TFF1 in vitro results in accelerated invasion of pancreatic cancer cells along with the characteristics of EMT. Additionally, experiments in genetically engineered mice show that the loss of TFF1 results in an EMT phenotype in PanIN and, eventually, in invasive adenocarcinoma. These data suggest that TFF1 functions as a tumor suppressor to inhibit EMT and the invasive transformation of PanIN. 
A

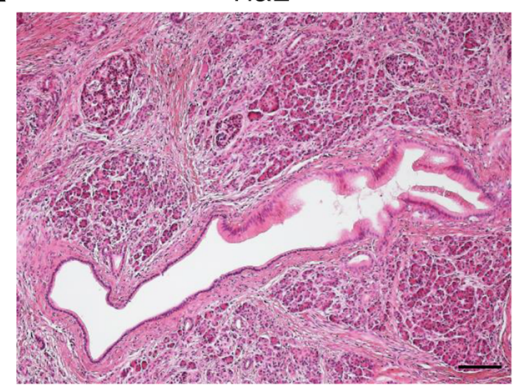

TFF1

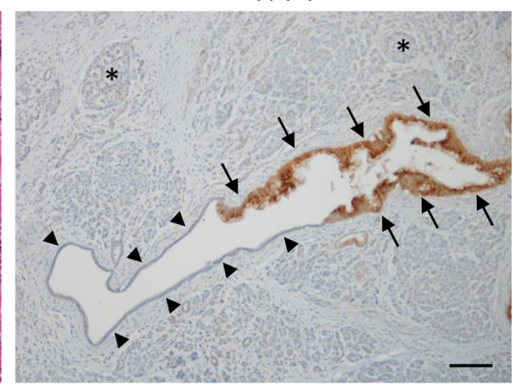

Figure 1. TFF1 expression is lost in the invasive component of pancreatic adenocarcinoma. (A) While abundant TFF1 expression was observed in PanIN (arrows), it was not observed in other pancreatic components, including acinar cells, islet cells (asterisks), and ductal epithelial cells (arrowheads). Scale bars: $100 \mu \mathrm{m}$. (B) The expression of TFF1 was confirmed in all grades of dysplasia. Scale bars: $50 \mu \mathrm{m}$. (C) TFF1 expression was observed in the intraductal component of PDAC and was lost in the invasive component. Scale bars: $200 \mu \mathrm{m}$ (left panel); $50 \mu \mathrm{m}$ (middle and right panels).

B

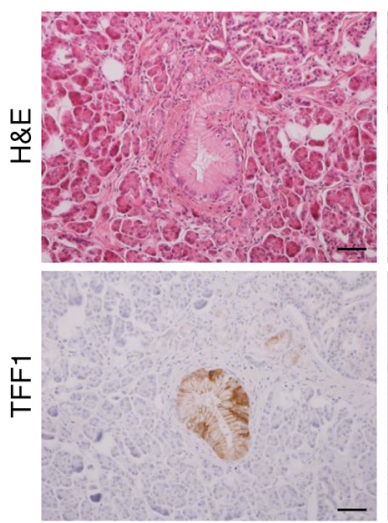

C

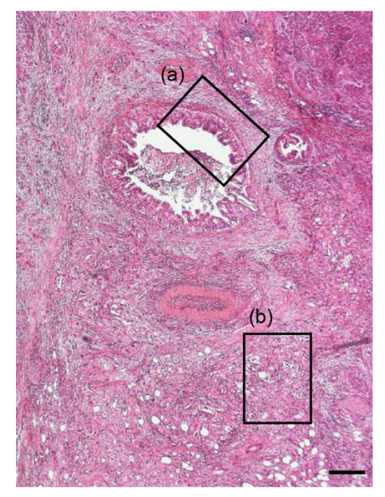

PanIN-2

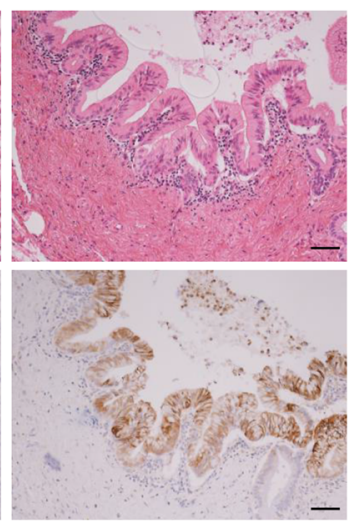

a. Intraductal component

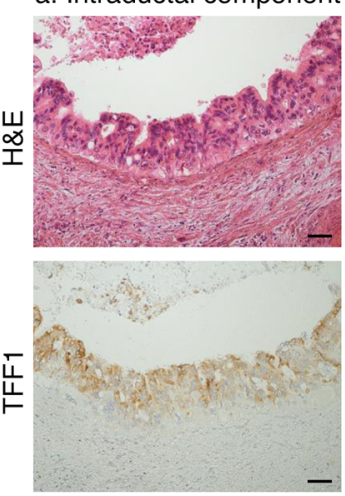

PanlN-3

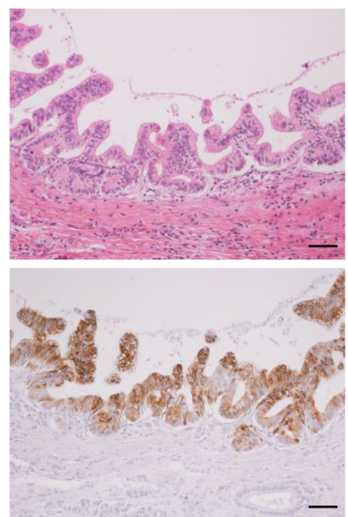

b. Invasive component

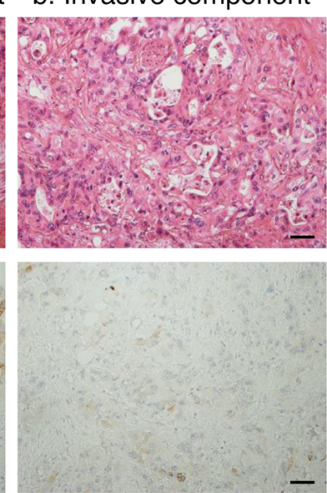

grade lesions. These findings suggest that the acquisition of TFF1 expression is an early event in pancreatic carcinogenesis. Next, the TFF1 expression in PDAC was assessed. TFF1 expression was observed in the intraductal component, but was frequently lost in the invasive component (Figure 1C), suggesting that TFF1 expression was lost during pancreatic carcinogenesis. Invasive IPMN (also described as PDAC derived from IPMN) also showed loss of TFF1 in the invasive component (Supplemental Figure 2B). Loss of TFF1 in the invasive component was observed in $50 \%$ of PDAC specimens $(n=7 / 14, P=0.016)$ and $72.7 \%$ of invasive IPMN specimens $(n=8 / 11, P=0.008$ ) (Supplemental Table 1), indicating that loss of TFF1 is associated with the invasive transformation of pancreatic neoplasms.

Suppression of TFF1 accelerates the invasion and migration of pancreatic cancer cell lines in vitro. To investigate the association between cancer invasion and loss of TFF1 in vitro, 2 pancreatic cancer cell lines, panc1 and PK9, were transfected with 3 different siRNAs against TFF1. A Boyden chamber assay revealed that both cell lines showed significantly upregulated invasion after TFF1 suppression (Figure 2, A and B). Conversely, the proliferative ability of both cell lines was not significantly different between the control and

\section{Results}

TFF1 is abundantly expressed in premalignant lesions of the pancreas, but is lost in invasive cancer. To investigate the contributions of TFF1 in pancreatic carcinogenesis, an IHC analysis of TFF1 was performed in surgically resected pancreatic specimens. As previously described (23), TFF1 expression was not found in normal pancreatic cells, such as acinar cells, islet cells, and pancreatic ductal epithelial cells (Supplemental Figure 1; supplemental material available online with this article; https://doi.org/10.1172/ JCI97755DS1), but some pancreatic duct glands (PDGs) showed faint TFF1 expression. In contrast, TFF1 was abundantly expressed in premalignant pancreatic lesions, such as PanIN (Figure 1A). Notably, extensive TFF1 expression was observed in PanIN-1 (low-grade dysplasia), PanIN-2 (moderate dysplasia), and PanIN-3 (high-grade dysplasia) lesions (Figure 1B). Similarly to PanIN, IPMN expressed a remarkable level of TFF1 (Supplemental Figure 2A), which was also found in both low-grade and high-
siRNA groups (Figure 2C). Next, the migration ability of pancreatic cancer cell lines was assessed by a wound-healing scratch assay, which revealed that the migration of cancer cells was significantly enhanced after TFF1 suppression in both cell lines (Figure 2, D and E). These results show that loss of TFF1 is not involved in cancer proliferation, but does enhance the invasive ability and migration of pancreatic cancer cells.

Loss of TFF1 results in EMT of pancreatic cancer cells. EMT is a process by which cancer cells gain migratory and invasive properties. Based on the hypothesis that TFF1-suppressed cancer cells undergo EMT and obtain invasive ability, EMT characteristics were assessed in siRNA-treated cancer cell lines. EMT is induced by several regulators, such as zinc finger transcription factors, including Snail/SNAI1, Slug/SNAI2, and Twist/TWIST1; thus, the expression of these proteins was assessed in these cells 4 days after transfection of siRNA. As expected, the expression levels of these proteins were upregulated in siRNA-treated cells (Figure 3A). The RNA expression of SNAI1 
A

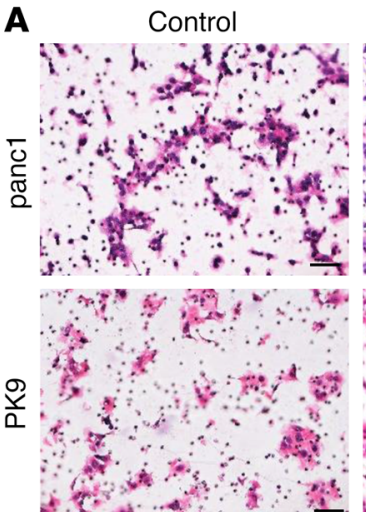

siTFF1-1

siTFF1-2

siTFF1-3
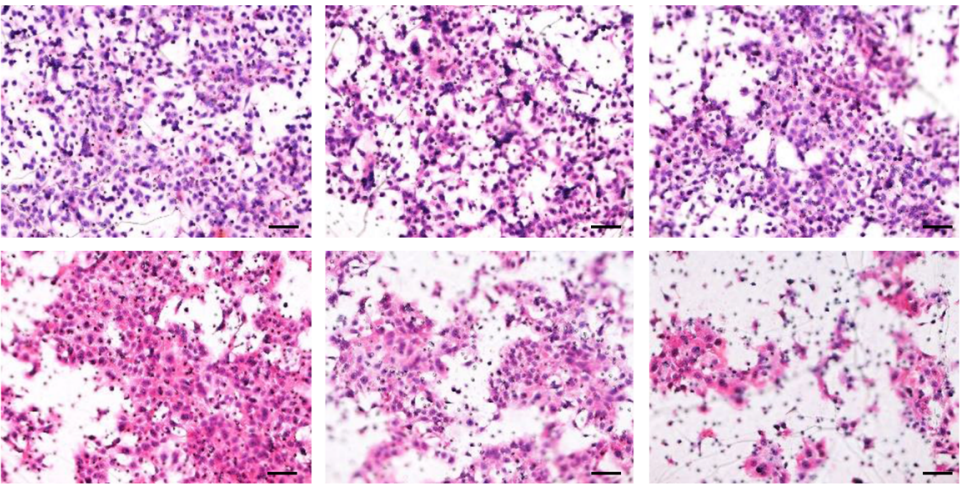

B

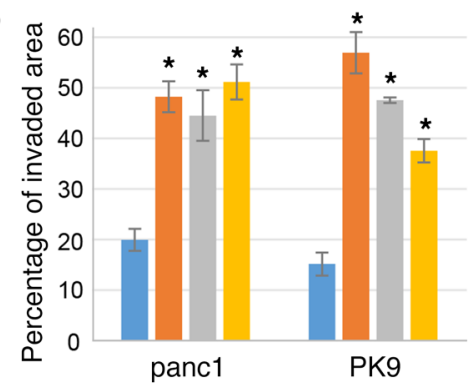

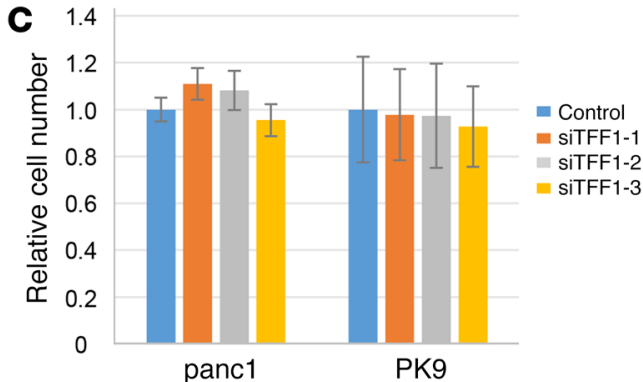

After migration

D
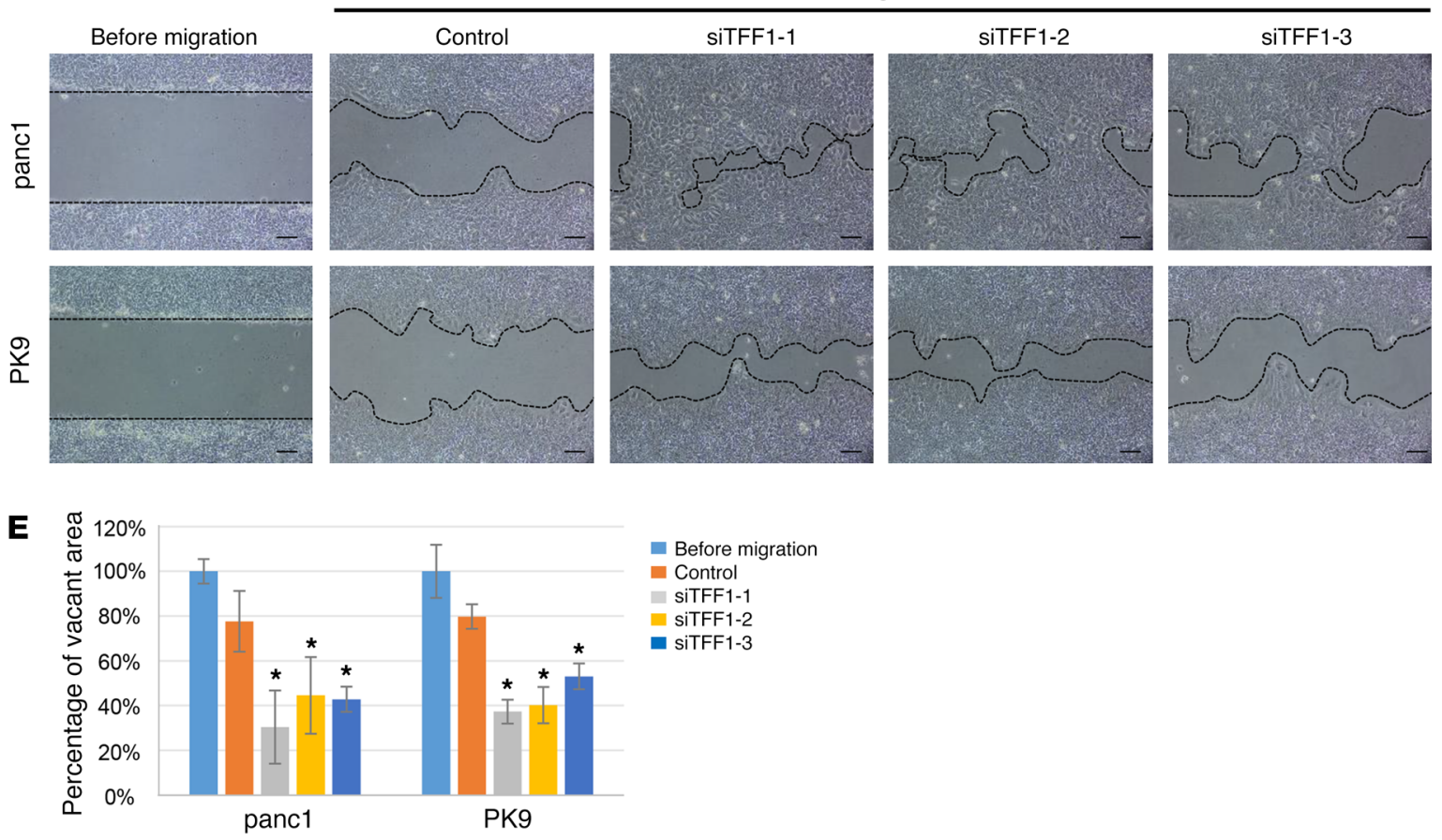

Figure 2. Suppression of TFF1 results in enhanced invasion and migration of pancreatic cancer cells. (A) A Boyden chamber assay revealed that cancer cells in the TFF1-suppressed group had high invasive ability. Scale bars: $50 \mu \mathrm{m}$. (B) Quantitative analysis of the Boyden chamber assay. ${ }^{*} P<0.01$, ANOVA. (C) Proliferation of cancer cells was assessed by a WST assay, which showed no significant differences in relative cell number (by ANOVA). (D) The woundhealing scratch assay revealed that the migration of cancer cells was significantly enhanced by the suppression of TFF1. Scale bars: $50 \mu \mathrm{m}$. (E) Quantitative analysis of the scratch assay. ${ }^{*} P<0.01$, ANOVA. Data are presented as mean \pm SD.

and SNAI2 was significantly upregulated, by 1.5 - to 3 -fold, when TFF1 was suppressed by siTFF1-1, -2, and -3 (Figure 3C), although a significant upregulation of TWIST1 was found only with siTFF1-2. A fundamental event in EMT is the process by which cells lose their polarity and cell-cell adhesions. The expression of cell-celladhesion molecules, such as E-cadherin/CDH1, occludin/OCLN, and $\mathrm{Zo}-1 /$ tight junction protein 1 (TJP1) was assessed. As expected, loss of TFF1 resulted in the suppression of these cell-adhesion mole- 
A

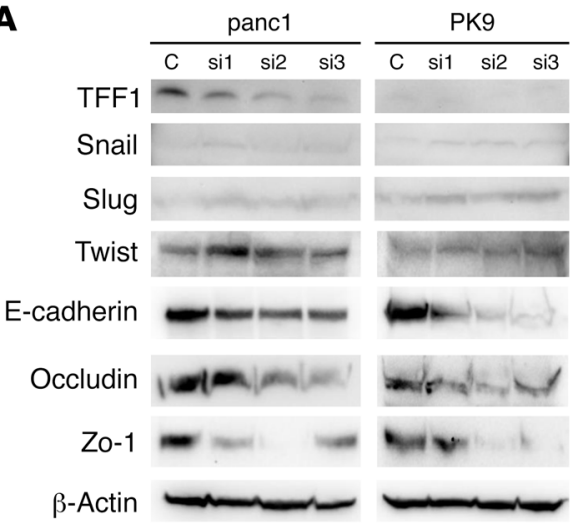

B
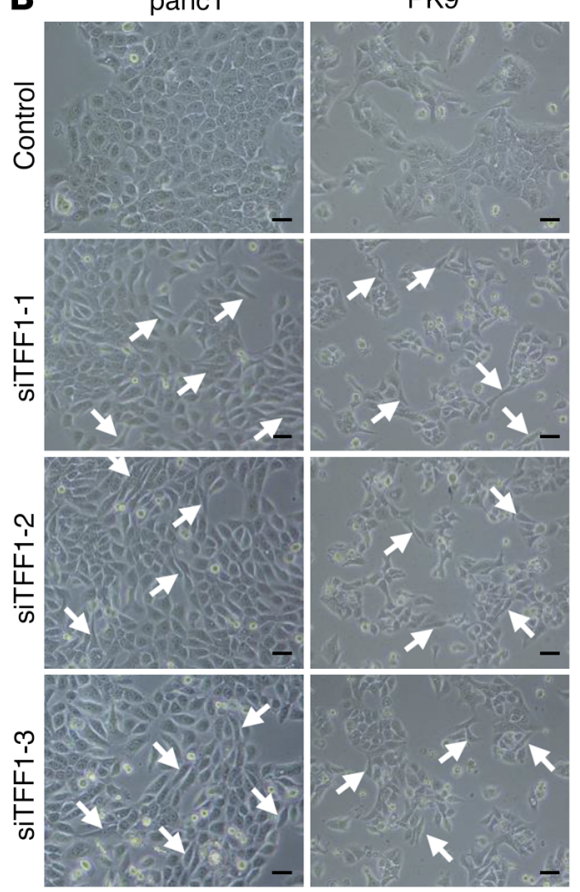

C
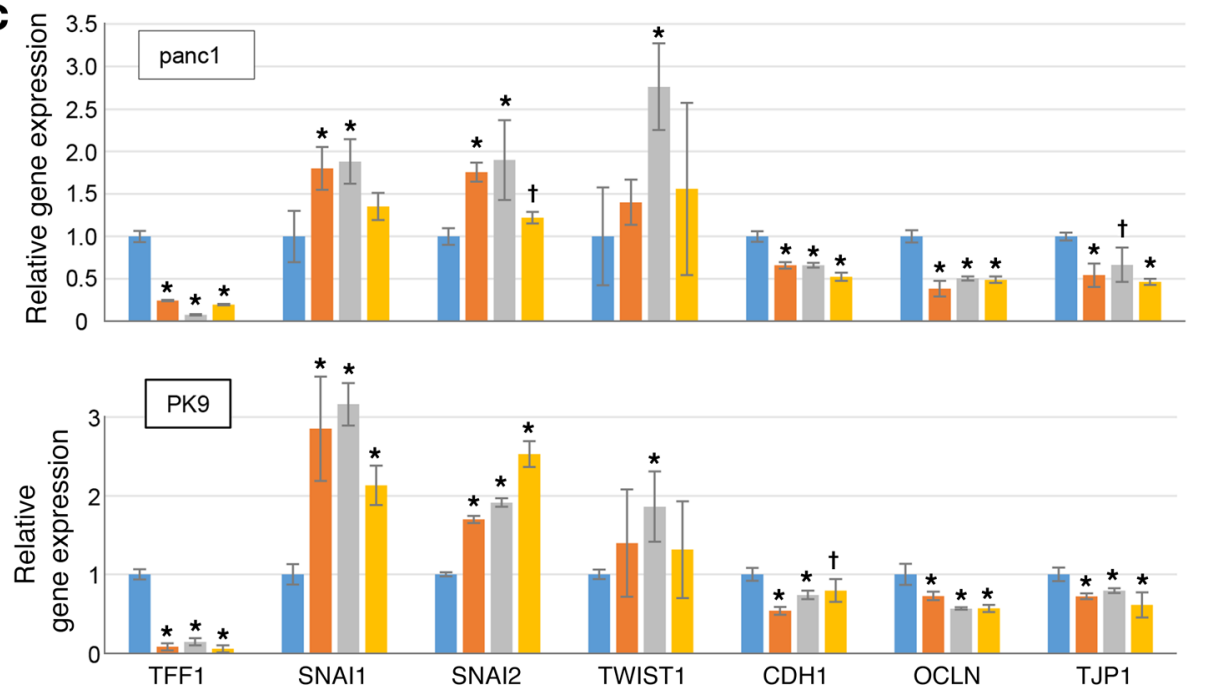

Figure 3. Suppression of TFF1 results in EMT of pancreatic cancer cells. (A) Western blotting revealed that the suppression of TFF1 caused an upregulation of Snail, Slug, and Twist, while downregulation of E-cadherin, Occludin, and Zo-1 was observed. $\beta$-Actin served as an internal control. (B) Suppression of TFF1 caused the cancer cells to exhibit a spindle-shaped morphology (arrows). Scale bars: $20 \mu \mathrm{m}$. (C) The realtime PCR results correlated to the protein expression levels induced by the suppression of TFF1. (D) Quantitative analysis of the percentage of spindle cells shown in $\mathbf{B}$. Data are presented as mean $\pm \mathrm{SD}$. ${ }^{*} P<0.01$; ${ }^{\dagger} P<0.05$, ANOVA.

Alternate expression of TFF1 and Snail in pancreatic tumor cells. Given that TFF1 is closely associated with cell migration, we evaluated the association between TFF1 expression and on the differences in confluency of cancer cells. As expected, the expression of TFF1 was significantly altered, depending on the confluence (Supplemental Figure 3). To investigate the relationship between TFF1 and zinc finger transcription factors in cancer cells, 8 pancreatic cancer cell lines (panc1, PK9, PK45h, PK59, KLM1, MiaPaca2, KP4, and PK8) were employed. The expression of TFF1 and zinc finger factors was evaluated when the cells became nearly confluent by real-time PCR, revealing that the expression levels of TFF1 and SNAI1 were inversely correlated (Figure $4 \mathrm{~A} ; r=-0.738, P=0.037$ ), while expression levels of SNAI2 and TWIST1 did not show a significant correlation with that of TFF1 (data not shown). These data suggest that Snail/SNAI1 is the zinc finger factor most closely correlated with TFF1 expression. Interestingly, the inverse correlation of TFF1 and SNAI1 was also observed in the same cells, depending on their confluence $(r=-0.92, P<0.001$; Supplemental Figure 3). Of note, 4 cell lines (panc1, PK9, PK45h, and PK59) had higher TFF1 than SNAI1 expression, while the other cules at both protein and RNA levels (Figure 3, A and C). In addition, the cells showed a spindle-like morphology in the TFF1-suppressed group (Figure 3, B and D), suggesting that TFF1 suppression leads to morphological changes in cancer cells from an epithelial to a mesenchymal phenotype. These results suggest that the loss of TFF1 in cancer cells results in EMT, eventually inducing the migration and invasive transformation of pancreatic cancer cells.
4 cell lines (KLM1, MiaPaca2, KP4, and PK8) showed higher SNAI1 expression than TFF1 expression. The protein expression of TFF1 and Snail showed a similar expression pattern (except for KLM1, with predominant TFF1 expression). These data indicate that the first 4 cell lines (panc1, PK9, PK45h, and PK59) might represent an epithelial phenotype, while the latter 3 cell lines (MiaPaca2, KP4, and PK8) represent an EMT phenotype of pancreatic can- 

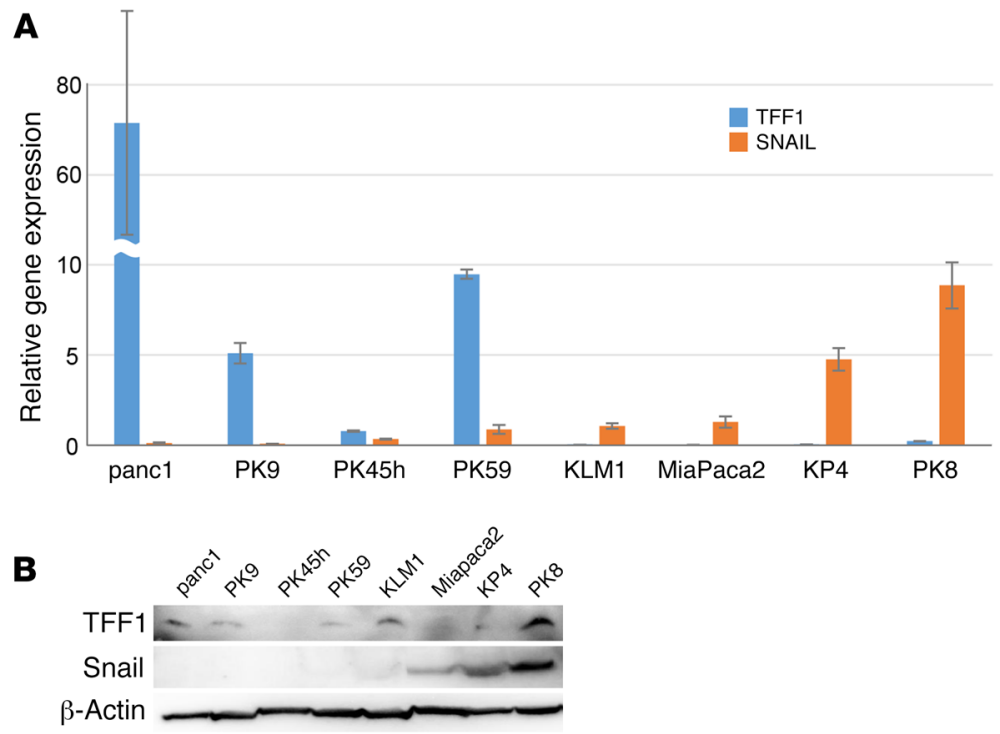

C
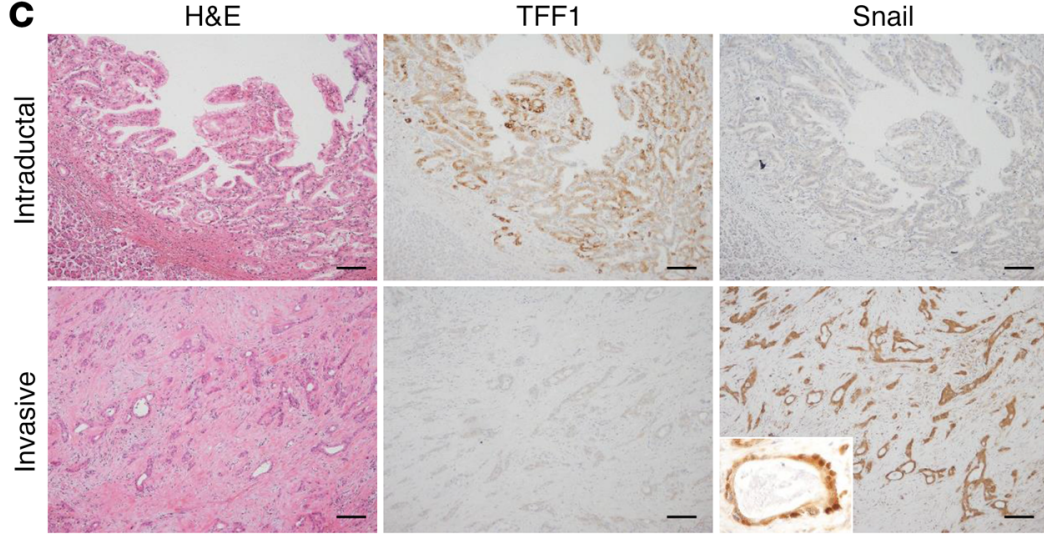

D

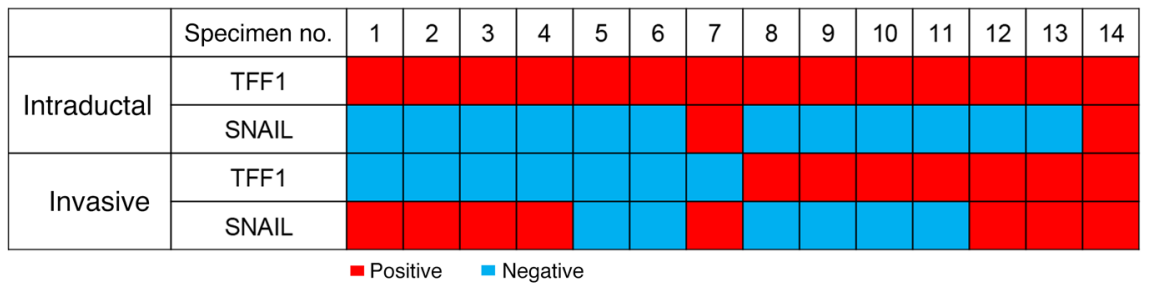

Figure 4. The contradictory expression of TFF1 and Snail in pancreatic cancer cells. (A) Realtime PCR revealed that 4 pancreatic cancer cell lines had higher TFF1 expression (panc1, PK9, PK45h, and PK59) than SNAl1 and 4 other lines had higher SNAl1 expression than TFF1 (KLM1, MiaPaca2, KP4, and PK8). The expression of TFF1 and Snail showed a significant inverse correlation. $r=-0.738 ; P=0.037$, Spearman's test. (B) Western blotting revealed a similar expression pattern for TFF1 and Snail. (C) The intraductal component of PDAC with strong TFF1 expression showed weak Snail expression, whereas the invasive component with weak TFF1 expression showed strong SNAIL expression. Inset: highmagnification image showing nuclear staining of Snail. Scale bars: $50 \mu \mathrm{m}$. (D) Heatmap of TFF1 and Snail expression in the PDAC specimen. An inverse correlation of TFF1 and Snail was also confirmed, $P=0.023$, by Pearson's $\chi^{2}$ test. Data are presented as mean \pm SD.

mens, in which the intraductal component strongly expressed TFF1 and the invasive component expressed Snail (Supplemental Figure 5; $P=0.006$ ). These data suggest that the loss of TFF1 is associated with the upregulation of Snail in the invasive component of human pancreatic tumors.

The phenotypes of $K C$ mice and $T F F 1^{-1-}$ mice. To evaluate the effect of the loss of TFF1 in vivo, Pdx-1 Cre; LSL-KRAS ${ }^{G 12 D}$ mice (KC mice, a model of pancreatic cancer) and TFF1-KO mice were employed. Consistent with previous reports $(24,25), \mathrm{KC}$ mice sacrificed at the age of 6 months and 12 months were found to have low-grade PanIN lesions in their pancreata, which never developed into PDAC ( $n=5$ and 10, respectively). The expression of TFF1 in the pancreata of KC mice was assessed by IHC, revealing that PanIN lesions in KC mice show abundant TFF1 expression (Figure 5A). No other components of the pancreas showed expression

cer cells. To further evaluate the relationship between TFF1 and Snail, Snail-predominant cancer cells (KP4 and PK8) were treated with 3 different siRNAs against Snail (Supplemental Figure 4). As a result, siRNA-1 and -2 successfully suppressed Snail/SNAI1 expression in both cell lines; however, the expression of TFF1 was not affected by these treatments. These data indicate that TFF1 is an upstream regulator of Snail.

To confirm the involvement of TFF1 in the expression of Snail, Snail expression was assessed in the human samples by IHC. In PDAC specimens, TFF1 and Snail expression levels were significantly inversely correlated $(P=0.023)$, with the intraductal component with strong TFF1 expression showing no Snail expression, while the invasive component showed strong Snail, but weak TFF1, expression (Figure 4, C and D). Expression patterns opposite of those of TFF1 and Snail were also found in IPMN speci- of TFF1 (Supplemental Figure 1). This expression pattern of TFF1 in the pancreas is the same as our findings in human samples, supporting the reproducibility of human pancreatic carcinogenesis in genetically engineered mice. TFF1-KO mice were purchased from the International Mouse Phenotyping Consortium (IMPC) (26). The schematic of this "knockout-first" allele of TFF1-KO is shown in Supplemental Figure 6A. This allele contains a lacZ trapping cassette and a floxed promoter-driven neo cassette inserted into the intron of a gene, disrupting gene function. Cre protein should delete the promoter-driven selection cassette and floxed exon of the allele. To confirm the function of this allele, the stomachs of these mice were harvested and assessed. IHC revealed that the surface of the gastric mucosa in WT mice expressed TFF1, while that of $\mathrm{TFF}^{-/-}$did not (Supplemental Figure 6, B and C). In the stomachs of $\mathrm{TFF}^{+1 /-}$ mice, the gastric mucosa surface showed 
A
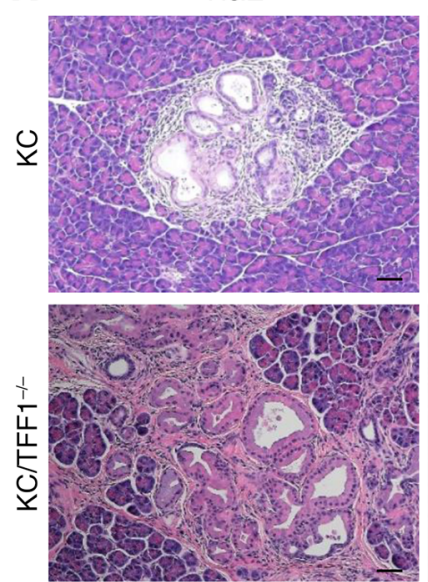

B
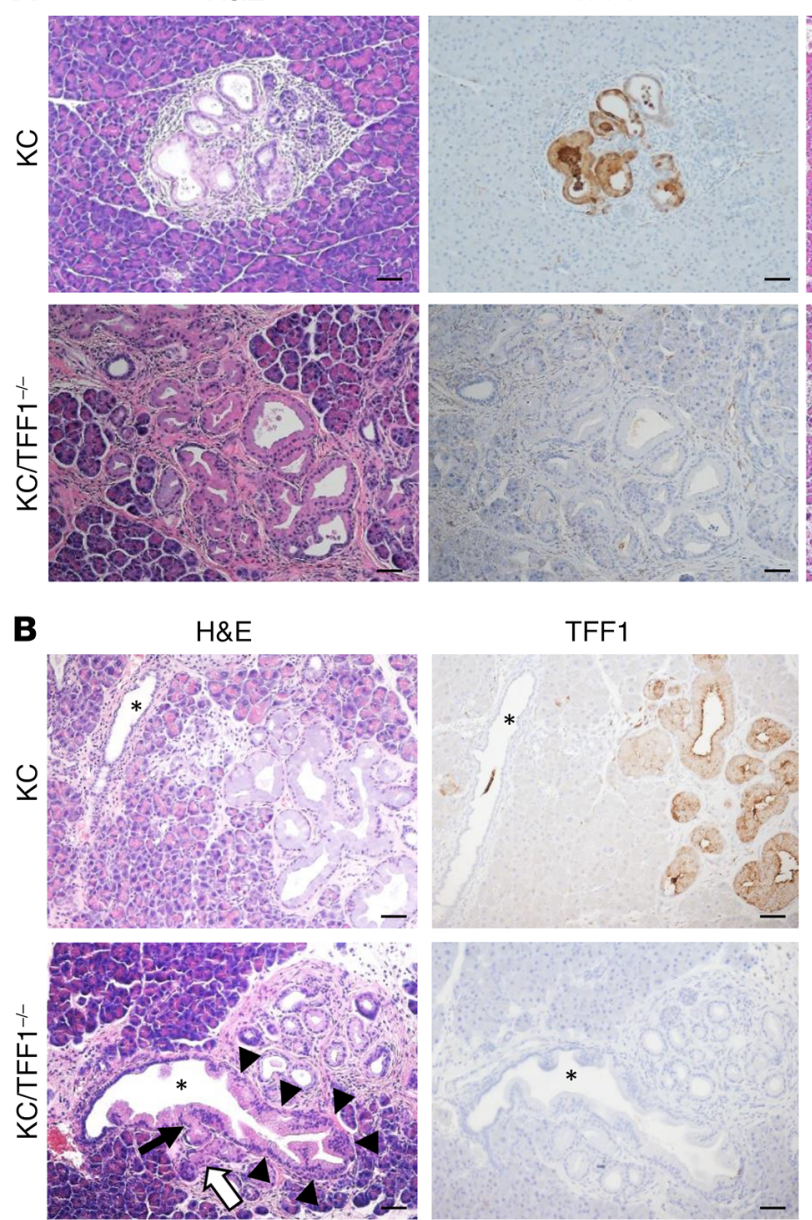

C

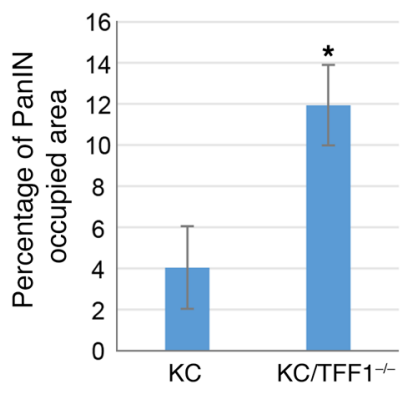

TFF1
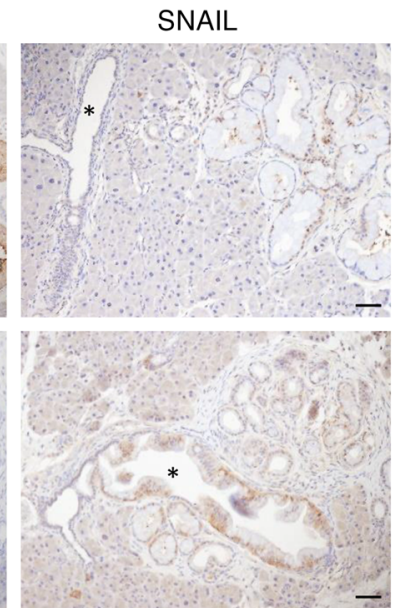

H\&E (low magnification)
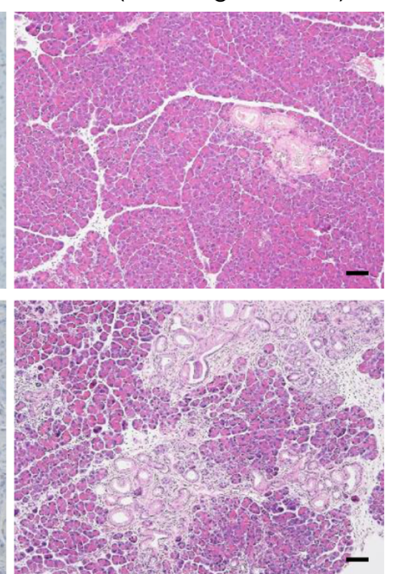

SNAIL

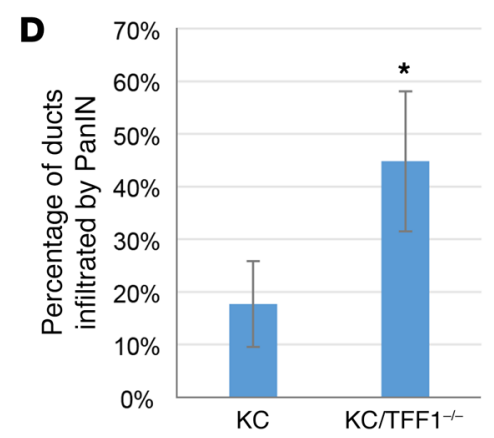

Figure 5. Loss of TFF1 results in the expansion of PanIN lesions in KC mice. (A) IHC revealed abundant TFF1 expression in PanIN cells in KC mice, while no expression was observed in PanlN cells in $\mathrm{KC} / \mathrm{TFFF}^{-1-}$ mice. In the low-magnification image, PanIN lesions were observed more extensively in $\mathrm{KC} / \mathrm{TFF}^{-1-}$ than in KC mice. Scale bars: 50 $\mu \mathrm{m}$ (left and middle panels); $100 \mu \mathrm{m}$ (right panels ). (B) PanIN lesions infiltrated into the ductal epithelium in $\mathrm{KC} / \mathrm{TFF}^{-1-}$, but not $\mathrm{KC}$, mice. PanIN cells without TFF1 expression were likely derived from acinar cells (white arrow), infiltrating into the pancreatic ducts (black arrow) and eventually spreading along pancreatic ducts and replacing normal ductal epithelial cells with tumor cells (black arrowheads) with strong Snail expression. Asterisks indicate ductal lumen. Scale bars: $50 \mu \mathrm{m}$. (C) Quantitative analysis of PanIN-occupied areas. ${ }^{*} P<0.01$, ANOVA. (D) Quantitative analysis of ducts infiltrated by PanIN lesions revealed more frequent infiltration in $\mathrm{KC} / \mathrm{TFF}^{-/-}$mice. ${ }^{*} P<0.01$, ANOVA. Data are presented as mean \pm SD.

(Figure 5C). Interestingly, PanIN cells without TFF1 expression infiltrated the pancreatic ducts, replacing cuboidal duct epithelial cells with mucinous PanIN cells with strong Snail expression in KC/ $\mathrm{TFF1}^{-/-}$mice (Figure 5B). The percentage of ducts infiltrated by PanIN lesions was significantly higher in $\mathrm{KC} / \mathrm{TFFF}^{-/-}$mice $(P<0.001)$, suggesting that the loss of TFF1 resulted in the migration of PanIN cells along with pancreatic ducts.

To further evaluate the impact of the loss of TFF1, the expression of Snail was assessed immunohistochemically. Snail expression in the pancreas was found only in the PanIN lesions and was classified into 3 categories based on the staining intensity: negative, weak, and strong (Figure 6A). Quantification of Snail

relatively weak TFF1 and lacZ expression (Supplemental Figure $6, \mathrm{D}$ and $\mathrm{E})$. No neoplastic lesions were found in the pancreata of 1-year-old TFF1 ${ }^{-/}$mice $(n=8)$.

Loss of TFF1 results in the expansion and EMT phenotype of PanIN in vivo. Based on the hypothesis that loss of TFF1 might transform the neoplastic characteristics of PanIN lesions in vivo, KC mice and $\mathrm{TFF1}^{--}$mice were bred to generate $\mathrm{KC} / \mathrm{TFF1}^{--}$mice. The phenotypes of KC $(n=5)$ and $\mathrm{KC} / \mathrm{TFF}^{-/-}(n=5)$ mice were assessed at the age of 6 months. IHC confirmed that PanINs in KC/TFF1 ${ }^{-1-}$ mice lacked expression of TFF1 (Figure 5A). The gross appearance of the pancreata was not significantly different between $\mathrm{KC}$ and $\mathrm{KC} / \mathrm{TFF}^{-/-}$mice; however, a histological examination showed that $\mathrm{KC} / \mathrm{TFF}^{-/-}$mice developed extensive areas occupied by PanIN, which were nearly 3-fold the size of those in KC mice expression revealed that PanIN in $\mathrm{KC} / \mathrm{TFF1}^{-/}$mice showed significantly stronger expression of Snail $(P<0.001$; Figure $6 \mathrm{~B})$. These observations suggest that loss of TFF1 induces EMT in PanIN cells and that mobilized PanIN cells migrate from the acinar area into ductal lesions, resulting in the expansion of PanIN-occupied areas.

Abundant cancer-associated fibroblasts were found in $\mathrm{KC} / \mathrm{TFF}^{-1-}$ mice. Given that the loss of TFF1 resulted in the EMT phenotype of tumor cells, we hypothesized that stromal cells could accumulate around the neoplastic lesions. To further evaluate the accumulation of stromal cells, cancer-associated fibroblasts (CAFs) were evaluated based on the expression of $\alpha$-smooth muscle actin $(\alpha$-SMA), a typical marker for CAFs. CAFs are associated not only with PDAC, but also with their precursor lesions, PanIN. To evaluate the involvement of CAFs in the PanIN areas, the expression of 
A

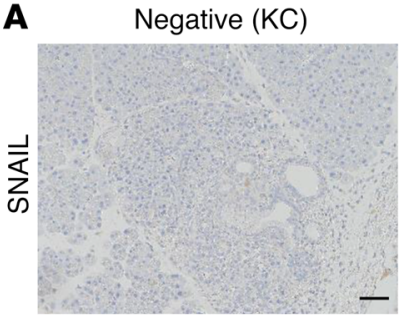

Weak (KC)

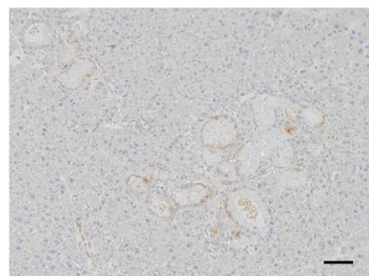

$-$

B

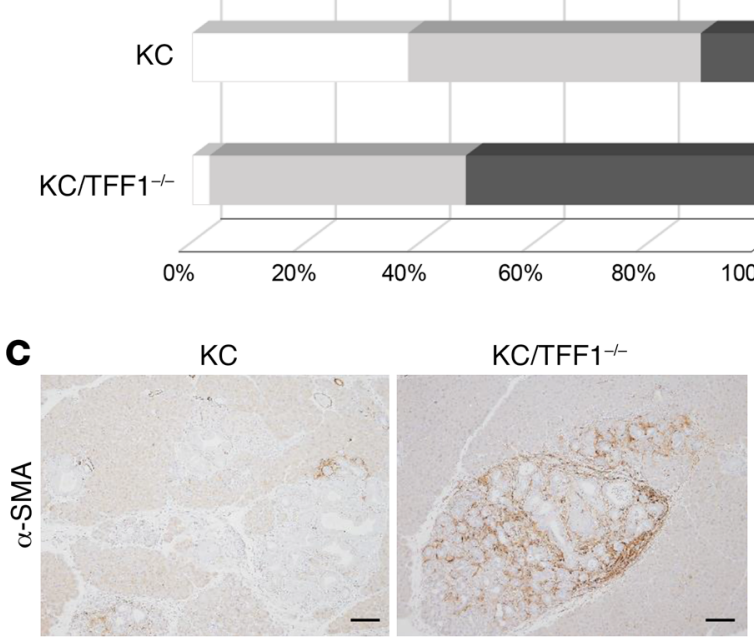

Strong $\left(\mathrm{KC} / \mathrm{TFF} 1^{---}\right)$

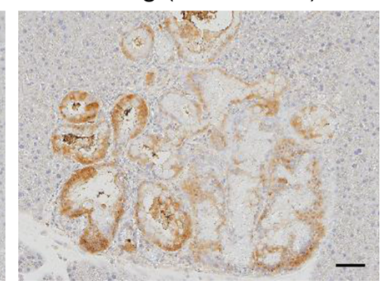

ONegative
C Weak

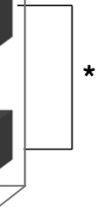

$\square$ Weak

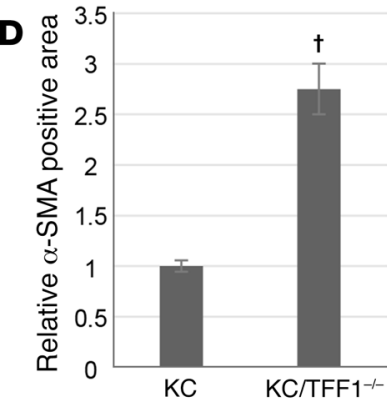

Figure 6. Loss of TFF1 induces an EMT phenotype in PanIN and accumulation of CAFs in KC mice. (A) Strong expression of SNAIL was observed in the PanIN cells of $\mathrm{KC} / \mathrm{TFF}^{-1-}$ mice. Scale bars: $50 \mu \mathrm{m}$. (B) Quantitative analysis of SNAIL expression. ${ }^{*} P<0.01$, ANOVA. (C) Strong expression of $\alpha$-SMA was observed in the PanIN areas of $\mathrm{KC} / \mathrm{TFF}^{-1-}$ mice. Scale bars: $50 \mu \mathrm{m}$. (D) Quantitative analysis of the $\alpha$-SMA-positive areas. ${ }^{\dagger} P<0.05$, ANOVA. Data are presented as mean \pm SD.

histological tumor features are equivalent to poorly differentiated adenocarcinoma in humans. Immunohistochemically, the tumors were composed of a CK19-positive epithelial component (Figure 8D) and abundant $\alpha$-SMA-positive fibroblasts (Figure $8 \mathrm{E}$ ). Substantial BrdU incorporation was observed in the tumor cells (Figure $8 \mathrm{~F})$. These findings indicate that loss of TFF1 results in the malignant and invasive transformation of PanIN, with extensive accumulation of CAFs.

To further identify the malignant potential of these microscopic tumors, the mice were followed up to the age of 12 months. While KC mice $(n=10)$ developed no malignant tumors in their pancreata,

$\alpha$-SMA in 6-month-old $\mathrm{KC}$ and $\mathrm{KC} / \mathrm{TFF}^{-/-}$mice was assessed by IHC. Extensive $\alpha$-SMA expression was found in the PanIN areas of $\mathrm{KC} / \mathrm{TFF}^{-/-}$mice compared with those of $\mathrm{KC}$ mice $(P=0.015$; Figure 6, C and D), indicating that loss of TFF1 caused the accumulation of CAFs, thus contributing to tumor formation.

Loss of TFF1 results in high-grade PanIN in KC mice. To evaluate the effect of the loss of TFF1 in pancreatic carcinogenesis, the grades of PanIN lesions found in 12-month-old $\mathrm{KC}$ and $\mathrm{KC} / \mathrm{TFF}^{-1-}$ mice were evaluated ( $n=10$ and 9, respectively). While most PanIN lesions found in KC mice were PanIN-1, PanIN-2 was found to spread across the pancreas in $\mathrm{KC} / \mathrm{TFF}^{-/-}$mice, and the percentages of PanIN-2 were higher in $\mathrm{KC} / \mathrm{TFF}^{-/-}$mice $(P<0.001$; Figure 7, $\mathrm{A}$ and $\mathrm{C}$ ). PanIN-3 lesions did not exist in $\mathrm{KC}$ mice, but were found in $2 \mathrm{KC} / \mathrm{TFF}^{-/}$mice. To further evaluate the malignant potential of PanINs, BrdU incorporation was assessed by IHC, which revealed that BrdU-positive cells were more frequently found in $\mathrm{KC} / \mathrm{TFF}^{-/-}$mice $(P=0.009$; Figure $7, \mathrm{~B}$ and $\mathrm{D})$. These data suggest that the malignant potential of PanIN increases in settings of TFF1 deficiency.

Loss of TFF1 on a background of KRAS activation results in the development of invasive pancreatic adenocarcinoma. Although tumors were not detected in the pancreas macroscopically, histological examination revealed that $80 \%$ of 6 -month-old $\mathrm{KC} /$ $\mathrm{TFF}^{-/-}$mice $(n=4 / 5)$ had solitary microscopic tumors in their pancreata (Figure 8A). The tumors were composed of cells with severe nuclear atypia, showing features of adenocarcinoma, with scattered gland formation (Figure 8B). The tumor cells had infiltrated into the surrounding parenchyma (Figure $8 \mathrm{C}$ ). These
$\mathrm{KC} / \mathrm{TFF}^{-/-}$mice $(77.8 \%, n=7 / 9)$ were found to have multiple microscopic tumors (the number of tumors ranged from 2 to 4 ), with histological features similar to those of the poorly differentiated adenocarcinomas found in 6-month-old $\mathrm{KC} / \mathrm{TFF}^{-/-}$mice (Supplemental Figure 7A). In addition, KC mice with heterozygous loss of TFF1 (KC/TFF1 ${ }^{+-}$) developed macroscopic tumors in their pancreata $(80 \%, n=74 / 5$; Figure $8, \mathrm{G}-\mathrm{I}$, and Supplemental Figure 7B). The histological features of this pancreatic tumor were equivalent to those of moderately differentiated human pancreatic adenocarcinoma. A KC/ $\mathrm{TFF}^{+/-}$mouse displayed multiple metastases in the liver (Figure 8, J-L). These findings suggest that loss of TFF1 can cause the development of PDAC with the capacity to metastasize and that partial loss of TFF1 is sufficient for this malignant transformation. The survival data of $\mathrm{KC}(n=731)$, $\mathrm{TFF}^{-1-}$ $(n=750)$, and $\mathrm{KC} / \mathrm{TFF}^{-/-}$mice $(n=733)$ were analyzed (Figure $8 \mathrm{M})$. Control $\mathrm{KC}$ and $\mathrm{TFF}^{-/-}$mice showed favorable survival, suggesting that loss of TFF1 alone or KRAS activation was not sufficient for the development of pancreatic malignancies. In contrast, $\mathrm{KC} / \mathrm{TFF}^{-/-}$mice exhibited a significantly worse survival time than control KC mice $(P=0.039)$, confirming the malignant potential of the tumors found in $\mathrm{KC} / \mathrm{TFF}^{-1-}$ mice.

\section{Discussion}

In this study, we investigated the expression of TFF1 in human samples and revealed that the acquisition of TFF1 expression is an early event of pancreatic carcinogenesis. Loss of TFF1 was associated with early EMT of PanIN in a mouse model, resulting in the expansion of PanIN, accumulation of CAFs, and eventually, the 
A

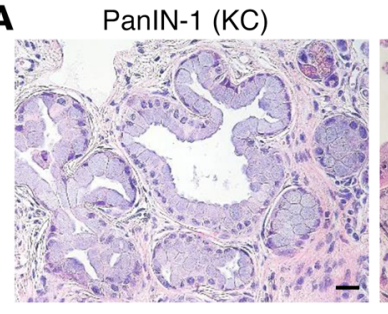

B

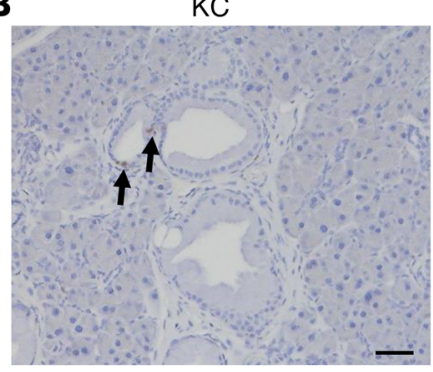

C

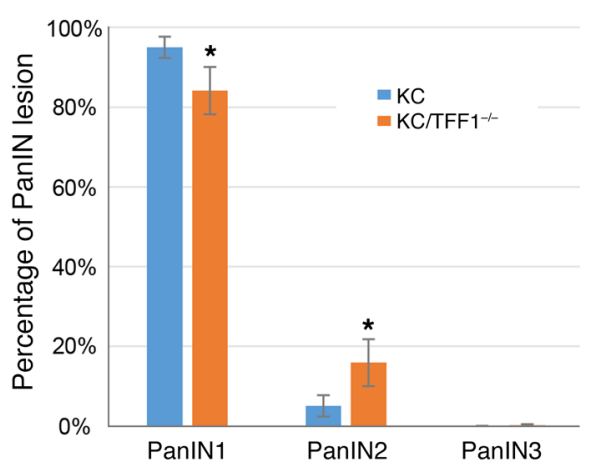

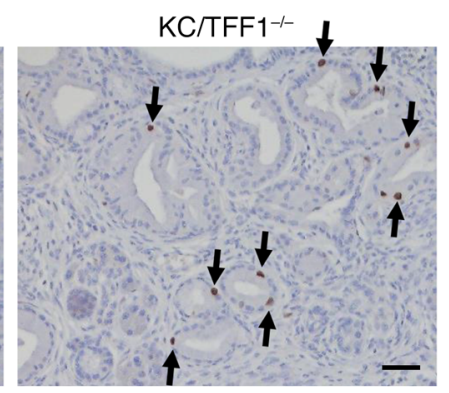

PanIN-2 (KC/TFF $\left.1^{--}\right)$

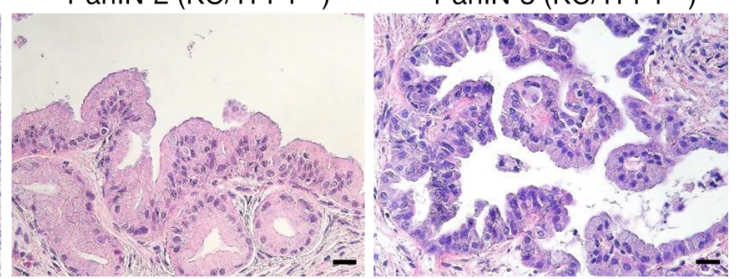

D

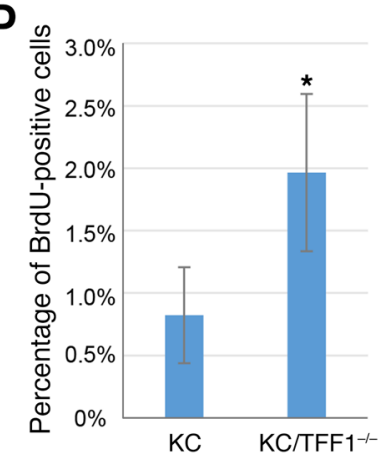

Figure 7. Loss of TFF1 results in high-grade dysplasia of PanIN lesions in KC mice. (A) Representative images of mouse PanINs. Scale bars: $20 \mu \mathrm{m}$. (B) IHC revealed high incorporation of BrdU (arrows) in PanIN cells in KC/TFF1 ${ }^{-/-}$mice. Scale bars: $50 \mu \mathrm{m}$. (C) Quantitative analysis of PanIN grading in mice. PanIN-2 frequency was significantly increased in $\mathrm{KC} / \mathrm{TFF}^{-1-}$ mice. ${ }^{*} P<0.01$, ANOVA. (D) Quantitative analysis of $\mathrm{BrdU}$ incorporation. Frequent $\mathrm{BrdU}$ incorporation was observed in PanIN cells in KC/ $\mathrm{TFF1}^{-/-}$compared with $\mathrm{KC}$ mice. ${ }^{*} P<0.01$, ANOVA. Data are presented as mean \pm SD.

ical aspect of EMT: while encouraging tumor invasion and metastasis, EMT can induce cell-cycle arrest $(34,35)$. Thus, acquisition of TFF1 expression in tumor cells might disrupt EMT of the cells, eventually resulting in the proliferation and enlargement of the tumor. In addition, our mouse model also showed that heterozygous loss of TFF1 resulted in macroscopic tumors in the pancreas, while complete loss merely caused microscopic tumors, suggesting that the complete loss of TFF1 might have induced extensive EMT and inhibited the proliferative ability of PDAC cells.

In terms of tumor initiation, the tissue stem cell niche and progenitor compartment are thought to be the origin of malignant disease (36). Strict control of cell regeneration is required in the stem cell niche; otherwise, the proliferation can be uncontrollable, eventu-

development of PDAC. These results indicate that TFF1 functions as a tumor suppressor that inhibits EMT and the invasive transformation of PanIN.

The role of TFF1 in malignant diseases has been elusive; some reports have suggested that TFF1 is a tumor-suppressor gene, while others have indicated that it is an oncogene. Recent studies have revealed that the expression of TFF1 is suppressed in gastric carcinogenesis in an epigenetic manner associated with Helicobacter infection (27) and that deficiency of TFF1 in mice is associated with gastric cancer $(17,28)$ and breast cancer $(29)$. Meanwhile, other studies have reported that TFF1 possesses oncogenic functions in breast (30) and pancreatic cancer cells $(31,32)$. Despite the inconsistencies among these reports, it should be noted that the tumor-suppressive role of TFF1 has been demonstrated by autochthonous mouse models in which the TFF1 gene is genetically deleted. Autochthonous models develop de novo tumors in the native microenvironment and can be used to represent the mechanisms of tumor initiation (33). Indeed, our mouse model of $\mathrm{KC} / \mathrm{TFF}^{-1-}$ recapitulates the initiation of PDAC from PanIN, thus implying the tumor-suppressive role of TFF1. In contrast, the oncogenic role of TFF1 has been shown in nonautochthonous mouse models in which cancer cell lines were treated with various vectors to suppress or increase the expression of TFF1 and then engrafted into host mice. The appearance of a tumor-promoting effect from TFF1 in these models might reflect the bilateral biolog- ally leading to the development of malignant diseases. Interestingly, the trefoil factors TFF1 and TFF2 are uniquely expressed in the progenitor compartment of the pancreatic ductal epithelium, PDGs (23), in the setting of inflammatory injury. We also previously revealed that TFF2 can function as a tumor suppressor to prevent the initiation of pancreatic premalignant lesions IPMN and PanIN (37). Taken together with the findings of this study, these results show that TFFs are likely key molecules not only in the regeneration of the pancreas, but also in inhibiting the initiation of pancreatic neoplastic lesions.

Although tumor dissemination has been thought to be the terminal event of cancer progression, recent studies have suggested that EMT can occur in the earliest stage of pancreatic carcinogenesis. Lineage tracing in genetic mouse models of PDAC revealed that invasive behavior and intravasation of tumor cells precede tumorigenesis (4) and that EMT can be observed even in PanIN lesions (3). Consistent with these findings, our mouse model also indicated that EMT could be induced in PanIN cells in $\mathrm{KC} / \mathrm{TFF}^{-/-}$mice, which could be partly explained by the expansion and Snail expression of PanIN cells. Interestingly, the consequence of early EMT is not only the invasive transformation of PanIN, but also the expansion of PanIN lesions and the accumulation of CAFs. Given the recent finding that the majority of PanINs are derived from acinar cells and not from ductal epithelium $(38,39)$, the PanINs in the ductal lesions of KC/ 
A
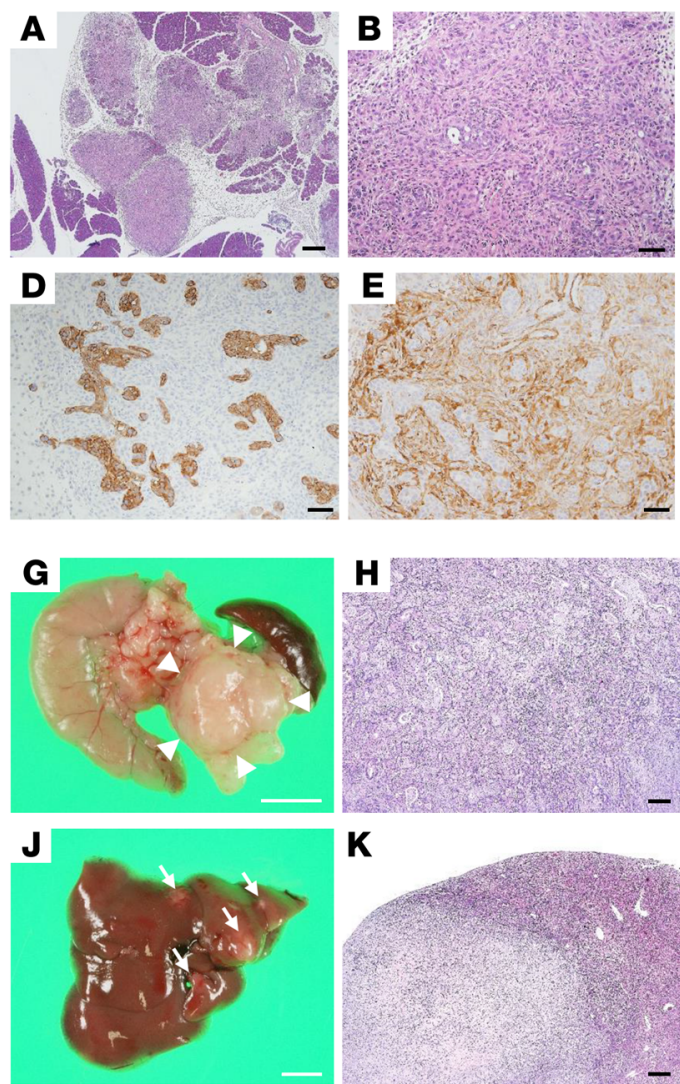

$\mathbf{K}$
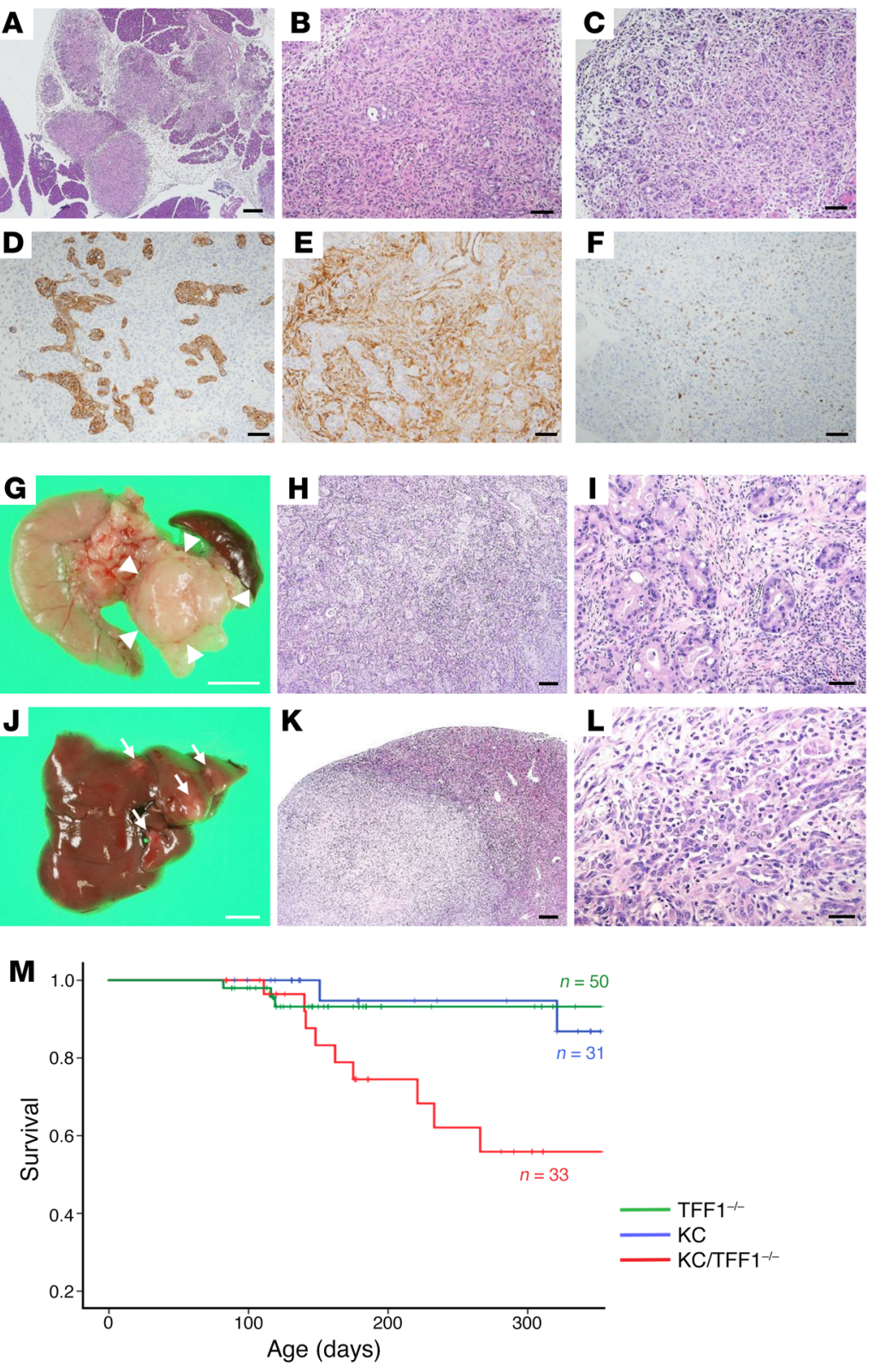

Figure 8. Loss of TFF1 results in the development of PDAC in $\mathrm{KC}$ mice. (A-F) A pancreatic tumor found in a $\mathrm{KC} / \mathrm{TFF}^{-1-}$ mouse. (A) A microscopic tumor found in a 6-month-old $\mathrm{KC} / \mathrm{TFF}^{-1-}$ mouse. (B) The tumor was equivalent to poorly differentiated human adenocarcinoma. (C) Tumor cells infiltrating the surrounding tissue. IHC revealed the expression of (D) CK19 and (E) $\alpha$-SMA as well as (F) BrdU incorporation in tumor cells. Scale bars: $200 \mu \mathrm{m}$ (A); $50 \mu \mathrm{m}$ (B-F). (G-L) Tumors found in a 12 -month-old $\mathrm{KC} / \mathrm{TFF}^{+/-}$mouse. (C) Gross appearance of a pancreatic tumor (arrowheads). ( $\mathbf{H}$ and $\mathbf{I})$ Histologically, the tumor exhibited the features of a moderately differentiated adenocarcinoma. (J) Gross appearance of multiple liver tumors (arrows). (K and $\mathbf{L}$ ) Multiple liver metastases were confirmed histologically. Scale bars: 5 mm (G and J); $200 \mu \mathrm{m}$ (H and $\mathbf{K}$ ); $50 \mu \mathrm{m}$ (I and $\mathbf{L}$ ). (M) Survival curves showing the significantly worse survival of $\mathrm{KC} \mathrm{TFF}^{-1-}$ (red) mice compared with KC (blue) and $\mathrm{TFF}^{-1-}$ (green) mice. $P=0.039$, log-rank test.

to be clarified by further investigation, including lineage tracing of PanIN cells in TFF1-KO mice to determine their fates.

The findings of this study emphasize the importance of TFF1 as a therapeutic target for PDAC. Recent studies have suggested that suppression of EMT can lead to enhanced sensitivity of pancreatic cancer to gemcitabine treatment $(41,42)$, indicating that TFF1 can inhibit EMT, thus attenuating drug resistance in PDAC. In addition, CAFs are thought to be crucial for the development of pancreatic cancer $(43,44)$ and for protecting pancreatic cancer cells against chemotherapy (45), also indicating that TFF1 treatment might reduce the accumulation of CAFs and eventually enhance the chemosensitivity of PDAC. Current combined chemotherapy for PDAC has increased PDAC patient survival, but the prognosis is still less than ideal $(46,47)$; thus, if TFF1 treatment was combined with these chemotherapies, their therapeutic effects on PDAC might be enhanced.

In conclusion, TFF1 functions as a tumor sup-

$\mathrm{TFF}^{-/-}$mice are likely the result of the EMT-induced migration of mobilized PanIN cells from the acinar component into pancreatic ducts, causing the expansion of PanIN lesions. Meanwhile, how CAFs accumulate in $\mathrm{KC} / \mathrm{TFF}^{-1-}$ mice is uncertain. If the extensive CAFs found in $\mathrm{KC} / \mathrm{TFF}^{-/}$mice were derived from preexisting fibroblasts, they might be the result of the proliferation or migration of fibroblasts. Given that TFF1 is a secreted protein, it is reasonable to assume that the lack of TFF1 in PanIN lesions resulted in the migration and accumulation of fibroblasts. In contrast, studies have revealed that CAFs can be derived from tumor cells via EMT (40) and that PanIN cells have the ability to transform into mesenchymal cells that are indistinguishable from the surrounding stromal cells (3). Given this evidence, one can also assume that loss of TFF1 results in EMT of PanIN cells, transforming epithelial cells into stromal cells and eventually causing extensive CAF formation in PanIN areas. This hypothesis that CAFs can be derived from PanIN cells by the loss of TFF1 needs pressor to prevent EMT and invasive transformation of pancreatic neoplastic cells. Further efforts are needed to elucidate the precise mechanisms of TFF1 as a tumor suppressor and to establish novel clinical therapeutic approaches for treating PDAC patients by targeting TFF1.

\section{Methods}

Human samples. Surgically resected human samples of PDAC $(n=$ 14), noninvasive IPMNs $(n=10)$, and invasive IPMNs $(n=13)$ were obtained from pancreatectomy specimens.

Histology and IHC. Specimens were fixed overnight in 10\% formalin/PBS. Histological analysis was performed on $4 \mu \mathrm{m}$ paraffin-embedded sections. IHC was performed using the antibodies shown in Supplemental Table 2 . The sections were incubated overnight at $4^{\circ} \mathrm{C}$ with each primary antibody and incubated for 1 hour at room temperature with secondary antibodies. Proteins were visualized using EnVision Detection Systems (Dako). Slides were counterstained with hematoxylin. 
Cell culture. Human pancreatic cancer cell lines (panc1, MiaPaca2, KLM1, KP4, PK8, PK9, PK45h, and PK59) were obtained from the Cell Resource Center for Biomedical Research (Institute of Development, Aging and Cancer, Tohoku University, Japan). All cell lines were cultured in RPMI 1640 medium (Invitrogen, Life Technologies), except for MiaPaca2, which was cultured in DMEM (Sigma-Aldrich), with $10 \%$ heat-inactivated FBS (Equitech-Bio Inc.) at $37^{\circ} \mathrm{C}$ in a humidified atmosphere with $5 \% \mathrm{CO}_{2}$.

Transfection of siRNA. Three human TFF1 siRNAs were chemically synthesized by Sigma-Aldrich. The sequences of the strands were as follows: TFF1 siRNA-1, sense: 5'-CUGGUGCUUCUAUCCUAAUTT-3', antisense: 5'-AUUAGGAUAGAAGCACCAGTT-3'; TFF1 siRNA-2, sense: 5'-GCUUCUAUCCUAAUACCAUTT-3', antisense: 5'-AUGGUAUUAGGAUAGAAGCTT-3'; TFF1 siRNA-3, sense: 5'-CUGUUUCGACGACACCGUUTT-3', antisense: 5'-AACGGUGUCGUCGAAACAGTT-3'; SNAI1 siRNA-1, sense: 5'-GCCUUCAACUGCAAAUACUTT-3', antisense: 5'-AGUAUUUGCAGUUGAAGGCTT-3'; SNAI1 siRNA-2, sense: 5'-GCUCCUUCGUCCUUCUCCUTT-3', antisense: 5'-AGGAGAAGGACGAAGGAGCTT-3'; SNAI1 siRNA-3, sense: 5'-GAACCUGCGGGAAGGCCUUTT-3', antisense: 5'-AAGGCCUUCCCGCAGGUUCTT-3'. The control siRNA was synthesized by QIAGEN, with the sequence as follows: sense: 5'-UUCUCCGAACGUGUCACGUdTdT-3', antisense: 5'-ACGUGAGACGUUCGGAGAAdTdT-3'. Cancer cell lines were transfected with $25 \mathrm{nM}$ siRNA using a CUY21EDIT ver 2.10 electroporation system (BEX) according to the manufacturer's protocol.

Boyden chamber assay. Invasion assays were performed using Boyden chambers containing a polycarbonate membrane $(8.0 \mu \mathrm{m}$ pore size; Corning Inc.) coated with Matrigel (BD). Cells were transfected with siRNA on day 0 , and on day $2,2 \times 10^{5}$ cells (panc1) or $2 \times$ $10^{6}$ cells (PK9) were placed in the upper chamber with RPMI 1640 containing 1\% FBS, while the lower chamber was filled with RPMI containing $10 \%$ FBS. On day 3 , the cells that had passed through the membrane were fixed with methanol and stained with H\&E. The invaded areas were measured in 5 randomly selected fields using ImageJ software (NIH).

Cell proliferation assay. Cell growth was assessed using a WST1 cell proliferation assay system (Cell Proliferation Reagent WST-1; Roche). Cancer cell lines were transfected with TFF1 siRNA and then seeded in a 96 -well plate $\left(5 \times 10^{3}\right.$ cells per well). After 72 hours of incubation, the medium was replaced with fresh medium containing $10 \mu \mathrm{l}$ WST-1 reagent. Relative cell numbers were calculated by measuring the absorbance of each well at $450 \mathrm{~nm}$.

In vitro migration assay. The migration of cancer cells was assessed by the wound-healing scratch assay. Cells were transfected by siRNA and placed in 6-well plates. After the cells reached confluence, an artificial wound was created by manually scraping the confluent monolayer cells with micropipette tips. The dishes were incubated for 48 hours, and the status of the gap closure was observed. Ten images were randomly acquired and analyzed quantitatively by calculating the cellvacant area using Image J software.

Western blotting. Proteins were extracted from cultured cells using Laemmli sample buffer (Wako). The proteins were subjected to $10 \%$ or $15 \%$ SDS-polyacrylamide electrophoresis (Tris-glycine system, except for TFF1 detection in Tris-tricine system) and transferred onto PVDF membranes (Immobilon; Millipore). The membranes were incubated with primary antibodies against TFF1 (1:1,000, rabbit polyclonal; Cell
Signaling Technology, catalog 12419), E-cadherin (1:1,000, rabbit monoclonal; Cell Signaling Technology, catalog 3195), Snail (1:1,000, rabbit monoclonal; Cell Signaling Technology, catalog 3879), Slug (1:1,000, rabbit monoclonal; Cell Signaling Technology, catalog 9585), Zo-1 (1:1,000, rabbit monoclonal; Cell Signaling Technology, catalog 8193), Twist (1:1,000, rabbit polyclonal; Cell Signaling Technology, catalog 46702), Occludin (1:500, rabbit polyclonal; Abcam, catalog ab168986), and $\beta$-actin (1:5,000, mouse monoclonal; SigmaAldrich). An HRP-conjugated anti-rabbit IgG (1:1,000, Cell Signaling Technology) and an HRP-conjugated anti-mouse IgG (1: 1,000, Cell Signaling Technology) were used as the secondary antibodies. The protein expression was detected using Pierce Western Blotting Substrate (Thermo Fisher Scientific).

Real-time PCR. RNA was extracted from cell lines using a QIAcube (QIAGEN) according to the manufacturer's protocols. cDNA was synthesized using a High Capacity cDNA Reverse Transcription Kit (Applied Biosystems). Real-Time PCR assay was performed using a 7300 Fast Real-Time PCR System (Applied Biosystems). Each reaction was performed in a $10 \mu \mathrm{l}$ mixture containing TaqMan Universal PCR Master Mix (Applied Biosystems). The TaqMan probes and primers for TFF1 (assay Hs00907239_m1), SNAI1 (assay Hs00195591_m1), SNAI2 (assay Hs00161904_m1), TWIST1 (assay Hs01675818_s1), CDH1 (assay Hs01023895_m1), OCLN (assay Hs05465837_g1), TJP1 (assay Hs01551861_m1), and 18S (assay Hs99999901_s1) were obtained from Applied Biosystems. In each evaluation, the relative expression of the gene of interest was normalized to that of the $18 \mathrm{~S}$ internal control.

Animals. The Pdx1-Cre (catalog 014647) and LSL-KRAS ${ }^{\text {G12D }}$ (catalog 008179) mice were purchased from the Jackson Laboratory. TFF1-KO (KO first allele, allele name: Tff1 ${ }^{\text {tmla(EUCOMM)Wtsi }}$ mice were purchased from the International Mouse Phenotyping Consortium (IMPC) (26). Pdx1-Cre and LSL-KRAS ${ }^{\mathrm{G} 12 \mathrm{D}}$ mice were bred with TFF1$\mathrm{KO}$ mice to generate mice with the following genotype: KC; TFF1-KO $\left(\mathrm{KC} / \mathrm{TFF}^{-/-}\right)$. BrdU was injected intraperitoneally 2 hours before sacrificing these mice.

Statistics. All data are presented as mean \pm SD. Differences were tested for significance by ANOVA. For the categorical analysis, Pearson's $\chi^{2}$ test or McNemar's test was employed as appropriate. The correlation analysis was performed using Spearman's test. The survival of the mice was calculated using the Kaplan-Meier method, and differences in the survival curves were compared using the log-rank test. To exclude the possibility of non-cancer-related deaths, the mice that died before 10 weeks of age were excluded from this analysis. All statistical analyses were performed using the Statistical Package for the Social Sciences (SPSS) ver. 24. $P<0.05$ was considered statistically significant.

Study approval. Human samples were collected and analyzed in accordance with approval from the Institutional Review Board of Nagoya University. Subjects gave informed consent. All animal experiments were conducted in compliance with guidelines of the Institute for Laboratory Animal Research, Nagoya University Graduate School of Medicine. All human and animal studies were approved by the Nagoya University Review Board of Bioethics.

\section{Author contributions}

JY designed research studies, conducted experiments, acquired data, and wrote the manuscript. YY, TK, and AE analyzed data and provided reagents. TE and MN provided administrative support. 


\section{Acknowledgments}

This work was supported by the Japan Society for the Promotion of Science (JSPS) and Grants-in-Aid for Scientific Research (KAKENHI), grant numbers 26893109 and 17K10695.
Address correspondence to: Junpei Yamaguchi, Surgical Oncology, Nagoya University Graduate School of Medicine, 65 Tsurumai-cho, Showa-ku, Nagoya, Aichi, Japan 466-8550. Phone: 81.52.744.2222; Email: jumpei@med.nagoya-u.ac.jp.
1. Siegel RL, Miller KD, Jemal A. Cancer statistics, 2016. CA Cancer JClin. 2016;66(1):7-30.

2. Hanahan D, Weinberg RA. Hallmarks of cancer: the next generation. Cell. 2011;144(5):646-674.

3. Wang $\mathrm{L}$, et al. ATDC induces an invasive switch in KRAS-induced pancreatic tumorigenesis. Genes Dev. 2015;29(2):171-183.

4. Rhim AD, et al. EMT and dissemination precede pancreatic tumor formation. Cell. 2012;148(1-2):349-361.

5. Koorstra JB, Feldmann G, Habbe N, Maitra A. Morphogenesis of pancreatic cancer: role of pancreatic intraepithelial neoplasia (PanINs). Langenbecks Arch Surg. 2008;393(4):561-570.

6. Hruban RH, et al. An illustrated consensus on the classification of pancreatic intraepithelial neoplasia and intraductal papillary mucinous neoplasms. Am J Surg Pathol. 2004;28(8):977-987.

7. Augustin T, Vandermeer TJ. Intraductal papillary mucinous neoplasm: a clinicopathologic review. Surg Clin North Am. 2010;90 (2):377-398.

8. Katabi N, Klimstra DS. Intraductal papillary mucinous neoplasms of the pancreas: clinical and pathological features and diagnostic approach. J Clin Pathol. 2008;61(12):1303-1313.

9. Kanda M, et al. Presence of somatic mutations in most early-stage pancreatic intraepithelial neoplasia. Gastroenterology. 2012;142(4):730-733.e9.

10. Fritz $S$, et al. Global genomic analysis of intraductal papillary mucinous neoplasms of the pancreas reveals significant molecular differences compared to ductal adenocarcinoma. Ann Surg. 2009;249(3):440-447.

11. Longman RJ, et al. Coordinated localisation of mucins and trefoil peptides in the ulcer associated cell lineage and the gastrointestinal mucosa. Gut. 2000;47(6):792-800.

12. Madsen J, Nielsen O, Tornøe I, Thim L, Holmskov U. Tissue localization of human trefoil factors 1, 2, and 3. J Histochem Cytochem. 2007;55(5):505-513.

13. Taupin D, Podolsky DK. Trefoil factors: initiators of mucosal healing. Nat Rev Mol Cell Biol. 2003;4(9):721-732.

14. Nomura S, et al. Spasmolytic polypeptide expressing metaplasia to preneoplasia in H. felis-infected mice. Gastroenterology. 2004;127(2):582-594.

15. Kjellev S. The trefoil factor family - small peptides with multiple functionalities. Cell Mol Life Sci. 2009;66(8):1350-1369.

16. Feng $G$, et al. DNA methylation of trefoil factor 1 (TFF1) is associated with the tumorigenesis of gastric carcinoma. Mol Med Rep. 2014;9(1):109-117.

17. Soutto M, et al. Loss of TFF1 is associated with activation of NF- $\mathrm{kB}$-mediated inflammation and gastric neoplasia in mice and humans. JClin Invest. 2011;121(5):1753-1767.

18. Peterson AJ, et al. Helicobacter pylori infection promotes methylation and silencing of trefoil factor 2, leading to gastric tumor development in mice and humans. Gastroenterology. 2010;139(6):2005-2017.

19. Fox JG, et al. Accelerated progression of gastritis to dysplasia in the pyloric antrum of TFF2C57BL6 $\times$ Sv129 Helicobacter pylori-infected mice. Am J Pathol. 2007;171(5):1520-1528.

20. Basturk O, et al. Preferential expression of MUC6 in oncocytic and pancreatobiliary types of intraductal papillary neoplasms highlights a pyloropancreatic pathway, distinct from the intestinal pathway, in pancreatic carcinogenesis. Am J Surg Pathol. 2010;34(3):364-370.

21. Kim GE, et al. Aberrant expression of MUC5AC and MUC6 gastric mucins and sialyl Tn antigen in intraepithelial neoplasms of the pancreas. Gastroenterology. 2002;123(4):1052-1060.

22. Nagata K, et al. Mucin expression profile in pancreatic cancer and the precursor lesions. J Hepatobiliary Pancreat Surg. 2007;14(3):243-254.

23. Yamaguchi J, et al. Pancreatic duct glands (PDGs) are a progenitor compartment responsible for pancreatic ductal epithelial repair. Stem Cell Res. 2015;15(1):190-202.

24. Hingorani SR, et al. Preinvasive and invasive ductal pancreatic cancer and its early detection in the mouse. Cancer Cell. 2003;4(6):437-450.

25. Gidekel Friedlander SY, et al. Context-dependent transformation of adult pancreatic cells by oncogenic K-Ras. Cancer Cell. 2009;16(5):379-389.

26. Brown SD, Moore MW. The International Mouse Phenotyping Consortium: past and future perspectives on mouse phenotyping. Mamm Genome. 2012;23(9-10):632-640.

27. Tomita $\mathrm{H}$, et al. Inhibition of gastric carcinogenesis by the hormone gastrin is mediated by suppression of TFF1 epigenetic silencing. Gastroenterology. 2011;140(3):879-891.

28. Soutto $\mathrm{M}$, et al. Activation of $\beta$-catenin signalling by TFF1 loss promotes cell proliferation and gastric tumorigenesis. Gut. 2015;64(7):1028-1039.

29. Buache $\mathrm{E}$, et al. Deficiency in trefoil factor 1 (TFF1) increases tumorigenicity of human breast cancer cells and mammary tumor development in TFF1-knockout mice. Oncogene. 2011;30(29):3261-3273.

30. Amiry N, et al. Trefoil factor-1 (TFF1) enhances oncogenicity of mammary carcinoma cells Endocrinology. 2009;150(10):4473-4483.

31. Arumugam T, et al. Trefoil factor 1 stimulates both pancreatic cancer and stellate cells and increases metastasis. Pancreas. 2011;40(6):815-822.

32. Radiloff DR, Wakeman TP, Feng J, Schilling S, Seto E, Wang XF. Trefoil factor 1 acts to suppress senescence induced by oncogene activation during the cellular transformation process. Proc Nat Acad Sci U S A. 2011;108(16):6591-6596.

33. Le Magnen C, Dutta A, Abate-Shen C. Optimiz- ing mouse models for precision cancer prevention. Nat Rev Cancer. 2016;16(3):187-196.

34. Evdokimova V, Tognon C, Ng T, Sorensen PH. Reduced proliferation and enhanced migration: two sides of the same coin? Molecular mechanisms of metastatic progression by YB-1. Cell Cycle. 2009;8(18):2901-2906.

35. Lovisa S, et al. Epithelial-to-mesenchymal transition induces cell cycle arrest and parenchymal damage in renal fibrosis. Nat Med. 2015;21(9):998-1009.

36. White AC, Lowry WE. Refining the role for adult stem cells as cancer cells of origin. Trends Cell Biol. 2015;25(1):11-20.

37. Yamaguchi J, et al. Loss of Trefoil factor 2 from pancreatic duct glands promotes formation of intraductal papillary mucinous neoplasms in mice. Gastroenterology.2016;151(6):1232-1244.e10.

38. Habbe N, et al. Spontaneous induction of murine pancreatic intraepithelial neoplasia (mPanIN) by acinar cell targeting of oncogenic Kras in adult mice. Proc Natl Acad Sci U S A. 2008;105(48):18913-18918.

39. Kopp JL, et al. Identification of Sox9-dependent acinar-to-ductal reprogramming as the principal mechanism for initiation of pancreatic ductal adenocarcinoma. Cancer Cell. 2012;22(6):737-750.

40. Spector I, Zilberstein Y, Lavy A, Nagler A, Genin O, Pines M. Involvement of host stroma cells and tissue fibrosis in pancreatic tumor development in transgenic mice. PLoS One. 2012;7(7):e41833.

41. Zheng X, et al. Epithelial-to-mesenchymal transition is dispensable for metastasis but induces chemoresistance in pancreatic cancer. Nature. 2015;527(7579):525-530.

42. Arumugam T, et al. Epithelial to mesenchymal transition contributes to drug resistance in pancreatic cancer. Cancer Res. 2009;69(14):5820-5828.

43. Neesse A, Algül H, Tuveson DA, Gress TM. Stromal biology and therapy in pancreatic cancer: a changing paradigm. Gut. 2015;64(9):1476-1484

44. Nielsen MF, Mortensen MB, Detlefsen S. Key players in pancreatic cancer-stroma interaction: Cancer-associated fibroblasts, endothelial and inflammatory cells. World J Gastroenterol. 2016;22(9):2678-2700.

45. Hessmann E, et al. Fibroblast drug scavenging increases intratumoural gemcitabine accumulation in murine pancreas cancer. Gut. 2018;67(3):497-507.

46. Conroy T, et al. FOLFIRINOX versus gemcitabine for metastatic pancreatic cancer. $N$ Engl J Med. 2011;364(19):1817-1825.

47. Shridhar R, et al. Increased survival associated with surgery and radiation therapy in metastatic gastric cancer: a Surveillance, Epidemiology, and End Results database analysis. Cancer. 2013;119(9):1636-1642. 